\title{
MOTIVATING ACADEMIC LIBRARIANS: IMPLICATIONS OF MASLOW'S HIERARCHY OF NEEDS THEORY
}

Hessah ALASOUSI

Kuwait University, Kuwait

hessahmlis@gmail.com

Bibi ALAJMI

Kuwait University, Kuwait

info@bibialaimi.com 


\section{Introduction}

- Motivation is the management process of influencing behavior based on the knowledge of what makes people act,

$\diamond$ It is the process that arouses, energizes, directs, and sustains behavior and performance; the process of stimulating people to action a task and achieves a desired goal,

- Understanding the factors that contribute to individual behavior in the workplace can provide an organization's management with the theoretical basis upon which to initiate change and begin improvement. 
previous research on motivation aimed to focus on motivational determinants as a proper explanation of individual behavior. These studies' findings support the notion that individual behavior is influenced by salient factors underlying the attitudes and goals that give rise to actions. 


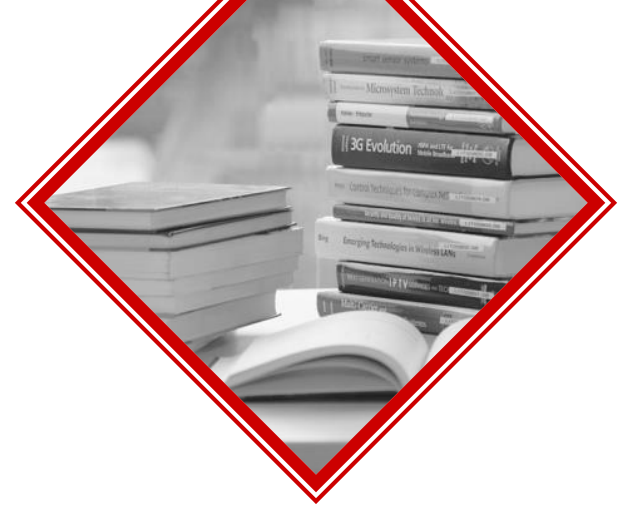

\section{Research Question}

What are the most effective factors that motivate librarians in Kuwait University's libraries according to Maslow's Needs Theory? 
Theoretical Framework: Maslow's Hierarchy of Needs Theory

Wisdom, Discernment, Understanding, and Context for Life; Self-fulfillment that can lead to a new focus on helping others (but not necessarily).

Competence, Approval, Status, Sense of Achievement

Mutual Social \& Intimate Relationships; Membership

Stability, Safety in family, society, \& one's organization

Survival \& Bodily Comfort

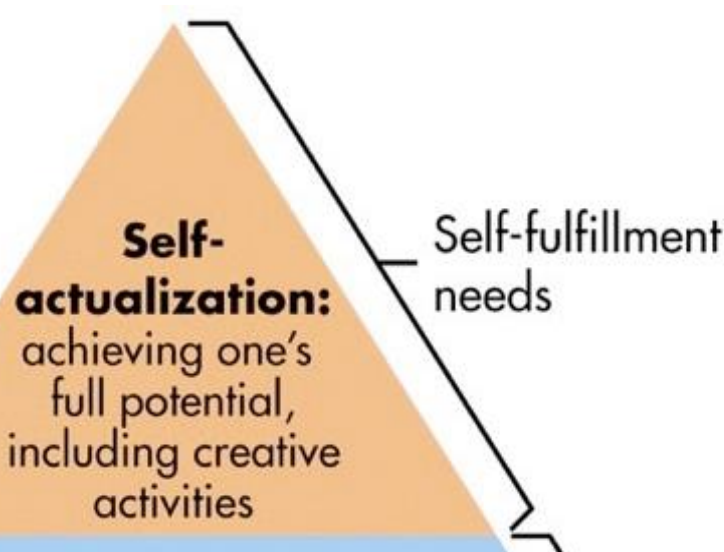

\section{Esteem needs:} prestige and feeling of accomplishment

Belongingness and love needs: intimate relationships, friends

\section{Safety needs: security, safety}

\section{Physiological needs:}

food, water, warmth, rest
Psychological needs

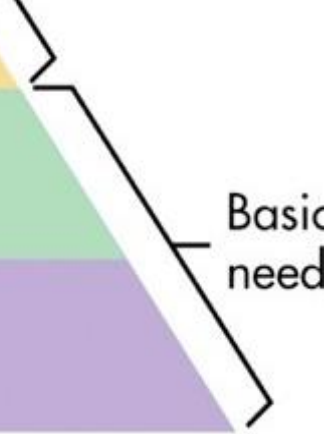




\section{Theoretical Framework: Maslow's Hierarchy of Needs Theory}

- Physiological needs: the basic needs of individuals, such as fresh air, an adequate workspace, well-designed workstations, comfortable lighting, suitable temperature, convenient food services, and water.

- Security needs: These can be satisfied by providing individuals with a safe office, stable wages and salaries, job security, health insurance, and retirement benefits.

- Social needs: To satisfy their social needs, particularly the need to love and be loved, employees need social activities, teamwork, friendship, a sense of belonging, and affection within the workplace.

- Esteem needs: Esteem needs may be divided into two categories. The first comprises the desire for strength, achievement, and adequacy, confidence in the face of the world, and independence and freedom. The second comprises the desire for reputation or prestige, recognition, attention, importance, or appreciation.

- Self-actualization needs: the desire for self-fulfillment, namely, the tendency to become actualized in what one has the potential to be. This tendency might be phrased as the desire to increasingly become what one is capable of becoming. 


\section{My Research Methodology}

\section{Instrument}

A survey was used as a primary tool for data collection
Sample

123 librarians in all 9

Kuwait University

colleges libraries

\section{Data Collection}

Surveys were sent and delivered to the librarians in all 9 libraries, and were collected after 5 working days with 61 returned surveys 


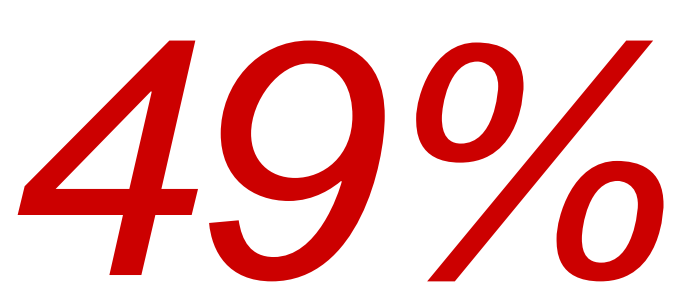

Response rate

$$
\text { w }
$$




\section{Responses were from each library}

Health Sciences Center $26 \%$
Jaber Al-Ahmad Library

$15 \%$
Library of Arts library of Sharea \& Islamic Studies

Library of Education

$11 \%$
Library of Life Sciences Library of Engineering and Petroleum

$10 \%$
Library of Science

Library of Law

$8 \%$ 


\section{First: Demographic Information}




\begin{tabular}{|c|c|c|c|}
\hline Demographic information & Dimension & Frequency & Percentage \\
\hline Gender & $\begin{array}{c}\text { Male } \\
\text { Female }\end{array}$ & $\begin{array}{l}18 \\
39\end{array}$ & $\begin{array}{l}32 \% \\
68 \%\end{array}$ \\
\hline Age & $\begin{array}{c}20-29 \\
30-39 \\
40-49 \\
50-59 \\
60 \text { above }\end{array}$ & $\begin{array}{c}6 \\
26 \\
16 \\
7 \\
1\end{array}$ & $\begin{array}{l}11 \% \\
46 \% \\
29 \% \\
13 \% \\
2 \%\end{array}$ \\
\hline Education & $\begin{array}{l}\text { High School } \\
\text { Diploma } \\
\text { University Degree } \\
\text { Graduate Degree }\end{array}$ & $\begin{array}{c}6 \\
6 \\
35 \\
9\end{array}$ & $\begin{array}{l}11 \% \\
11 \% \\
63 \% \\
16 \%\end{array}$ \\
\hline Years of Experience & $\begin{array}{c}\text { Less than } 2 \\
2-5 \\
6-10 \\
11-15 \\
16 \text { above }\end{array}$ & \begin{tabular}{c|}
2 \\
6 \\
14 \\
15 \\
19
\end{tabular} & \begin{tabular}{|l|}
$4 \%$ \\
$11 \%$ \\
$25 \%$ \\
$27 \%$ \\
$34 \%$
\end{tabular} \\
\hline
\end{tabular}




\section{Positions in the library}

\begin{tabular}{|l|c|c|}
\hline Position & Frequency & Percentage \\
\hline Librarian & 29 & $54 \%$ \\
\hline Other Positions & 15 & $28 \%$ \\
\hline Library assistant or Technician & 7 & $13 \%$ \\
\hline Library Director & 3 & $6 \%$ \\
\hline
\end{tabular}




\section{Second: Maslow's Levels of Needs}




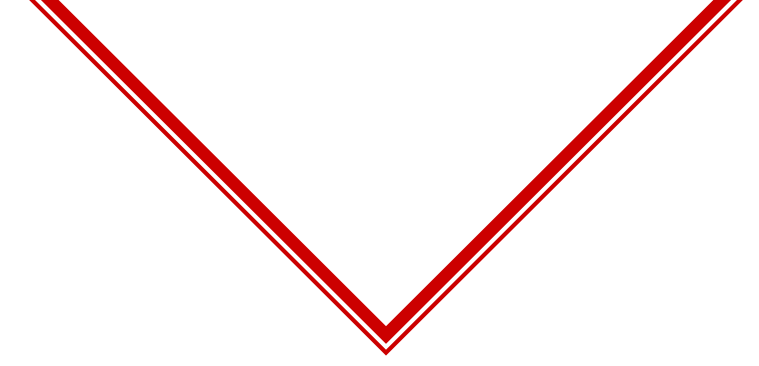

\section{Level of Motivation}


Table I: Motivation Levels

\begin{tabular}{l|l|l|c|c}
\hline No & \multicolumn{1}{|c|}{ Maslow's order } & Current Study's order & Mean & SD \\
\hline 1 & Physiological & Self-Actualization & 4.022 & .950 \\
\hline 2 & Security & Social Needs & 3.801 & 1.061 \\
\hline 3 & Social needs & Physiological & 3.738 & .979 \\
\hline 4 & Self-esteem & Self-Esteem & 3.393 & .871 \\
\hline 5 & Self-actualization & Security & 3.592 & .902 \\
\hline & & & & \\
\hline
\end{tabular}


- The empirical findings of this study do not support the concept of need hierarchy, nor it was meant to test it statistically.

Previous literature findings concluded while acknowledging that human beings do have needs to be met, the empirical evidence for the hierarchical order of the needs proposed by Maslow is sparse (Wahba and Bridwell, 1976);

The existence of a rigid order of needs for every individual is questioned (King-Hill, 2015). 


\section{Table II: Physiological Needs}

\begin{tabular}{c|l|c|c|c|c|c|c|c}
\hline & \multicolumn{1}{|c|}{ Needs } & $\mathbf{1}$ & $\mathbf{2}$ & $\mathbf{3}$ & $\mathbf{4}$ & $\mathbf{5}$ & $\mathbf{M}$ & SD \\
\hline 1 & $\begin{array}{l}\text { Office tools and stationery are sufficiently available } \\
\text { in my work environment }\end{array}$ & $8 \%$ & $15.2 \%$ & $10.6 \%$ & $33 \%$ & $33 \%$ & 3.69 & 1.28 \\
\hline 2 & $\begin{array}{l}\text { Modern technological equipment is sufficiently } \\
\text { available in my work environment }\end{array}$ & $3.7 \%$ & $15.6 \%$ & $9.2 \%$ & $34.9 \%$ & $36.7 \%$ & 3.84 & 1.19 \\
\hline 3 & $\begin{array}{l}\text { My work environment is comfortable for performing } \\
\text { my duties }\end{array}$ & $3.7 \%$ & $11.1 \%$ & $10.2 \%$ & $35.2 \%$ & $39.8 \%$ & 3.94 & 1.14 \\
\hline 4 & $\begin{array}{l}\text { Other features for comfortable work environment, } \\
\text { such as ventilation, lighting, air conditioning, } \\
\text { furniture, and other means, are sufficiently available }\end{array}$ & $5.4 \%$ & $9 \%$ & $11.7 \%$ & $38.7 \%$ & $35.1 \%$ & 3.89 & 1.12 \\
\hline 5 & $\begin{array}{l}\text { The library management pays enough attention to the } \\
\text { work environment }\end{array}$ & $10 \%$ & $10 \%$ & $15.5 \%$ & $30.9 \%$ & $33.6 \%$ & 3.67 & 1.32 \\
\hline 6 & $\begin{array}{l}\text { There are convenient and reasonable food facilities in } \\
\text { or near the library }\end{array}$ & $7.1 \%$ & $10.6 \%$ & $8.8 \%$ & $34.5 \%$ & $38.9 \%$ & 3.84 & 1.24 \\
\hline & Overall & & & & & & 3.738 & .979 \\
\hline
\end{tabular}




\section{Table III: Security Needs}

\begin{tabular}{c|l|c|c|c|c|c|c|c}
\hline & \multicolumn{1}{|c|}{ Needs } & $\mathbf{1}$ & $\mathbf{2}$ & $\mathbf{3}$ & $\mathbf{4}$ & $\mathbf{5}$ & $\mathbf{M}$ & $\mathbf{S D}$ \\
\hline 1 & $\begin{array}{l}\text { My workplace is free from hazards that } \\
\text { may cause injury }\end{array}$ & $3.6 \%$ & $12.6 \%$ & $9.9 \%$ & $36.9 \%$ & $36.9 \%$ & 3.87 & 1.15 \\
\hline 2 & I feel I am secure in my job & $4.5 \%$ & $4.5 \%$ & $8.2 \%$ & $30.9 \%$ & $51.8 \%$ & 4.17 & 1.08 \\
\hline 3 & $\begin{array}{l}\text { I feel peaceful and reassured every time I } \\
\text { go to work }\end{array}$ & $4.5 \%$ & $5.5 \%$ & $11.8 \%$ & $31.8 \%$ & $46.4 \%$ & 4.07 & 1.11 \\
\hline 4 & My employer provides health insurance & $45 \%$ & $10.8 \%$ & $14.4 \%$ & $9.9 \%$ & $19.8 \%$ & 2.42 & 1.57 \\
\hline & Overall & & & & & & 3.592 & .902 \\
\hline
\end{tabular}




\section{Table IV: Social Needs}

\begin{tabular}{c|l|c|c|c|c|c|c|c}
\hline \multicolumn{1}{|c|}{ Needs } & $\mathbf{1}$ & $\mathbf{2}$ & $\mathbf{3}$ & $\mathbf{4}$ & $\mathbf{5}$ & $\mathbf{M}$ & SD \\
\hline 1 & $\begin{array}{l}\text { I feel that I belong to the place where I } \\
\text { work }\end{array}$ & $10.3 \%$ & $6.5 \%$ & $12.1 \%$ & $31.8 \%$ & $39.3 \%$ & 3.82 & 1.28 \\
\hline 2 & $\begin{array}{l}\text { There is mutual trust between my boss and } \\
\text { me at work }\end{array}$ & $4.6 \%$ & $7.4 \%$ & $8.3 \%$ & $28.7 \%$ & $50.9 \%$ & 4.14 & 1.14 \\
\hline 3 & $\begin{array}{l}\text { The library encourages me to form good } \\
\text { relationships with my colleagues at work }\end{array}$ & $7.3 \%$ & $8.2 \%$ & $11.8 \%$ & $28.2 \%$ & $44.5 \%$ & 3.89 & 1.27 \\
\hline 4 & $\begin{array}{l}\text { The library encourages employees to work } \\
\text { on teams }\end{array}$ & $10.9 \%$ & $10.9 \%$ & $16.4 \%$ & $25.5 \%$ & $36.4 \%$ & 3.60 & 1.36 \\
\hline 5 & $\begin{array}{l}\text { I feel a sense of identity derived from } \\
\text { working at the library }\end{array}$ & $6.6 \%$ & $9.4 \%$ & $20.8 \%$ & $28.3 \%$ & $34.9 \%$ & 3.73 & 1.22 \\
\hline 6 & Co-workers help each other & $7.3 \%$ & $11.8 \%$ & $9.1 \%$ & $27.3 \%$ & $44.5 \%$ & 3.84 & 1.31 \\
\hline 7 & $\begin{array}{l}\text { My supervisor provides and foster a sense } \\
\text { of belongingness to the employees }\end{array}$ & $6.4 \%$ & $4.6 \%$ & $13.8 \%$ & $34.9 \%$ & $40.4 \%$ & 3.98 & 1.12 \\
\hline & Overall & & & & & & 3.801 & 1.061 \\
\hline
\end{tabular}




\section{Table V: Self-Esteem Needs}

\begin{tabular}{c|l|c|c|c|c|c|c|c}
\hline \multicolumn{1}{|c|}{ Needs } & $\mathbf{1}$ & $\mathbf{2}$ & $\mathbf{3}$ & $\mathbf{4}$ & $\mathbf{5}$ & $\mathbf{M}$ & SD \\
\hline 1 & $\begin{array}{l}\text { There is appreciation and mutual respect } \\
\text { between employees and their bosses at } \\
\text { work }\end{array}$ & $5.4 \%$ & $3.6 \%$ & $9.0 \%$ & $29.7 \%$ & $52.3 \%$ & 4.13 & 1.12 \\
\hline 2 & $\begin{array}{l}\text { My boss appreciates my work effort and } \\
\text { loyalty }\end{array}$ & $5.6 \%$ & $6.5 \%$ & $6.5 \%$ & $28.0 \%$ & $53.3 \%$ & 4.13 & 1.17 \\
\hline 3 & I feel satisfied about my job title & $10.9 \%$ & $7.3 \%$ & $12.7 \%$ & $36.4 \%$ & $32.7 \%$ & 3.68 & 1.29 \\
\hline 4 & $\begin{array}{l}\text { The library rewards employees' } \\
\text { achievements (e.g., promotional } \\
\text { allowances, medals, certificates, etc.) }\end{array}$ & $32.4 \%$ & $17.1 \%$ & $19.0 \%$ & $17.1 \%$ & $14.3 \%$ & 2.63 & 1.44 \\
\hline 5 & $\begin{array}{l}\text { The library holds an annual ceremony to } \\
\text { honor the employee of the year }\end{array}$ & $40.7 \%$ & $11.1 \%$ & $24.1 \%$ & $10.2 \%$ & $13.9 \%$ & 2.37 & 1.41 \\
\hline 6 & $\begin{array}{l}\text { I feel "wanted" for my professional } \\
\text { knowledge and competence }\end{array}$ & $10.3 \%$ & $7.5 \%$ & $15.0 \%$ & $37.4 \%$ & $29.9 \%$ & 3.67 & 1.26 \\
\hline & Overall & & & & & & 3.393 & .871 \\
\hline
\end{tabular}




\section{Table VI: Self-Actualization Need}

\begin{tabular}{c|l|c|c|c|c|c|c|c}
\hline & \multicolumn{1}{|c|}{ Needs } & $\mathbf{1}$ & $\mathbf{2}$ & $\mathbf{3}$ & $\mathbf{4}$ & $\mathbf{5}$ & $\mathbf{M}$ & SD \\
\hline 1 & $\begin{array}{l}\text { I consistently seek to help the library achieve } \\
\text { its goals }\end{array}$ & $3.6 \%$ & $1.8 \%$ & $11.8 \%$ & $34.5 \%$ & $48.2 \%$ & 4.20 & .989 \\
\hline 2 & $\begin{array}{l}\text { I consistently seek to properly employ my } \\
\text { skills in my job }\end{array}$ & $1.9 \%$ & $5.6 \%$ & $9.3 \%$ & $31.5 \%$ & $51.9 \%$ & 4.27 & .978 \\
\hline 3 & $\begin{array}{l}\text { I feel proud and honored to be performing my } \\
\text { work in this role }\end{array}$ & $4.5 \%$ & $3.6 \%$ & $11.6 \%$ & $23.2 \%$ & $57.1 \%$ & 4.21 & 1.10 \\
\hline 4 & $\begin{array}{l}\text { I constantly search for new opportunities to } \\
\text { learn in my field }\end{array}$ & $5.6 \%$ & $6.5 \%$ & $15.0 \%$ & $29.0 \%$ & $43.9 \%$ & 3.96 & 1.17 \\
\hline 5 & $\begin{array}{l}\text { My qualifications fit appropriately with the } \\
\text { nature of my work in the library }\end{array}$ & $6.3 \%$ & $8.9 \%$ & $12.5 \%$ & $31.3 \%$ & $41.1 \%$ & 3.88 & 1.21 \\
\hline 6 & $\begin{array}{l}\text { I feel self-fulfillment - that is, the feeling of } \\
\text { being able to use one's own unique } \\
\text { capabilities and realize one's potential }\end{array}$ & $4.5 \%$ & $8.2 \%$ & $19.1 \%$ & $21.8 \%$ & $46.4 \%$ & 3.96 & 1.19 \\
\hline & Overall & & & & & & 4.022 & .950 \\
\hline
\end{tabular}




\section{Key Findings}

- Results provides a clear distinction between different types of motivations that, once understood, could untangle the causes of human behavior and predict individuals' future actions.

Self-actualization, social needs, and (partially) self-esteem have shown strong evidence of motivation among academic library staff. 
Thanks!

Any question? 